\title{
Persone come noi: Intervista a Rita
}

Giornale di Tecniche Nefrologiche e Dialitiche 2019, Vol. 3I(I) 6I-64

(C) The Author(s) 2019

Article reuse guidelines:

sagepub.com/journals-permissions DOI: $10.1177 / 0394936219837880$ journals.sagepub.com/home/gtn

(S)AGE

\author{
Rita si racconta: dopo 20 anni in cerca di una diagnosi, \\ trova un aiuto dal web
}

\section{Rita, il tuo primo contatto "inconsapevole" con la malattia di Anderson-Fabry è avvenuto molti anni fa, quando ti sei imbattuta in una diagnosi di "cornea verticillata". Com'è successo? È stato fatto qualche approfondimento dopo la scoperta di questa particolarità alla tua cornea?}

All'epoca avevo 17 anni e una leggera miopia (era il 1975). Dover portare gli occhiali mi sembrava un dramma, così convinsi mio padre che dovevo assolutamente mettermi le lenti a contatto. Lui comprese la mia crisi adolescenziale e mi diede il suo benestare. La Dottoressa che si occupava di eseguire la visita oculistica, guardandomi il fondo dell'occhio con l'apparecchiatura apposita, si accorse stupita di una particolarità: la cornea in entrambi gli occhi non era trasparente, ma presentava una sorta di opacità a forma di stella. Mi invitò a recarmi alla clinica oculistica universitaria della mia città per esporre il mio caso. Quando il medico di turno mi visitò, confermò la diagnosi e chiamò altri medici oculisti che erano in quel momento in altre stanze. Mentre tutto questo succedeva, io me ne stavo con il mento appoggiato davanti alla loro apparecchiatura, ferma immobile a mostrare quel che, a mio avviso, mi faceva sentire molto speciale. Mi chiesero di fare delle foto per pubblicarle su dei testi di medicina oculistica ed io acconsentii. Questo confermava che ero proprio speciale! Purtroppo, molti videro le mie cornee, ma nessuno capì il vero problema e dissero che la causa dell'opacità era dovuta a depositi di accumulo. Più precisamente scrissero: "La cornea presenta una degenerazione verticillata la cui causa è sconosciuta; possibile una etiologia da accumulo ...". Erano gli anni' 80 e nessuno sospettava ancora cosa ci potesse essere sotto questo primo segnale di malattia. Mi feci fare le lenti a contatto facendo spendere a mio padre un bel po'di soldi e, con suo grande disappunto, non le portai che poche volte, dato che i miei occhi non le tolleravano.

\section{Purtroppo, le scarse conoscenze sulla malattia di Fabry non hanno permesso agli oculisti di scoprire la vera causa della tua cornea verticillata, anche se le loro intuizioni, da quello che racconti, non erano molto lontane! Oltre alla cornea verticillata non hai mai manifestato alcuna altra sintomatologia tipica della malattia di Fabry?}

Dopo i 12 anni di età, mi facevano tanto male le mani ed i piedi quando avevo la febbre o quando assumevo determinate posture. Ho avuto anche degli episodi di vertigini con svenimenti durante $\mathrm{i}$ quali sono stata ricoverata all'ospedale, inoltre la mia digestione mi ha dato grossi problemi fin da ragazza. Solo ora so che tutti questi problemi erano manifestazioni tipiche della malattia di Fabry. Poi, all'età di 52 anni ho iniziato ad avere continue extrasistoli che non smettevano né di giorno né di notte. Questo strano modo di comportarsi del mio cuore non mi permetteva di addormentarmi e di dormire bene, così mi sono recata al Pronto Soccorso. Là mi hanno ricoverata e i vari esami hanno denunciato una cardiomiopatia ipertrofica che da allora viene tenuta sotto controllo da farmaci beta-bloccanti e vengo sottoposta a controlli annuali.

Presidente Aiaf Onlus - Associazione Italiana Anderson-Fabry, Italy

Corrispondenza:

Stefania Tobaldini, Presidente Aiaf Onlus - Associazione Italiana

Anderson-Fabry, Italy.

Email: presidente@aiaf-onlus.org 
La malattia di Fabry, però, non ha coinvolto solo te, ma si è manifestata anche a tuo figlio, circa venti anni fa, quando era ancora piccolo. $\mathbf{C i}$ racconti come avete vissuto gli anni della sua infanzia?

Fin dall'età di sei anni mio figlio accusava forti dolori ai piedi e alle mani, disordini intestinali con conseguente anemia, e febbricola costante che non scendeva mai al di sotto dei $37.4^{\circ} \mathrm{C}$. Nei periodi di crisi, passava intere giornate senza alzarsi dal letto e un paio di volte é anche svenuto per il dolore. La sua prostrazione era una continua preoccupazione per me; anche il mal di testa pian piano ha cominciato a essere spesso presente. Non c'era tregua e sembrava non ci fosse soluzione alcuna.

Siamo andati avanti per anni, venti, venti lunghissimi anni senza che nessuno ci indicasse cosa avesse mio figlio o gli fosse venuto il minimo sospetto che la sua fosse una malattia genetica rara. Credo che chiunque abbia un figlio possa immaginare cosa prova un genitore in una situazione del genere, anche se, ringraziando il cielo, con il tempo i dolori si sono ridotti, non di molto ma sufficientemente da riuscire a gestirli con continue dosi di farmaci antidolorifici.

\section{Venti anni senza ricevere una risposta che giustificasse i dolori di tuo figlio sono veramente tanti! Come siete riusciti a gestire la vostra quotidianità?}

Come tutti i ragazzini del mondo anche mio figlio nella sua infanzia desiderava frequentare altri bambini, e fare qualche attività che gli permettesse di liberare quell'esuberanza fisica che si possiede in quel periodo di vita. Gli piaceva il calcio, così lo iscrissi in una squadra rionale dove con grande sua gioia infilò la divisa e si diresse dal Mister. La delusione arrivò presto insieme ai suoi dolori. I piedi arrossati gli scottavano e nonostante il suo entusiasmo, dovette rinunciare a frequentare la squadra. Pianse per la sua sfortuna e la delusione, ed io con lui, cercando però di non farmi vedere per non scoraggiarlo ulteriormente. Provammo allora con il judo, sperando che, non dovendo correre tanto, i suoi piedi non si sarebbero stancati. Ma niente da fare: i dolori pungenti non gli permisero neanche questo ripiego. I mali di pancia aumentarono, divenne anemico. Sembrava che tutto questo fosse causa di innumerevoli intolleranze alimentari così gli vennero tolti molti cibi e molti altri ancora perché c'era familiarità tra i vari gruppi di alimenti. Poteva mangiare solo poche cose ma la situazione non migliorava. Incominciò a perdere giorni di scuola perché non riusciva ad appoggiare i piedi per terra e così se ne stava giornate intere a letto tutto raccolto, per sentire meno il dolore. Gli davo la Tachipirina nelle giornate peggiori e si tirava avanti tra una visita ortopedica e l'altra con il risultato di sentirmi dire che erano "dolori di crescita".

Venne visto da molti medici specialisti in discipline diverse; lo visitarono anche dottori specialisti in medicine alternative e sinceramente devo dire che oltre agli antidolorifici, ciò che gli procurò un po' di riduzione del dolore, fu un rimedio omeopatico che usò per anni. Io ogni giorno mi scrivevo tutti i dati e le informazioni possibili e immaginabili: temperatura del corpo, pressione atmosferica, tempo meteorologico, cosa aveva mangiato a cena e a pranzo, come era andata la sua giornata a scuola, se aveva vissuto delle frustrazioni o meno, in modo da dare tutte le indicazioni al riguardo nel caso fossero servite. Sembravo paranoica, ma quando vivi una situazione del genere devi trovare una soluzione per aiutare la tua creatura che a causa del suo stare a letto tutto teso e ripiegato in se stesso, incominciava a sviluppare una brutta scoliosi. Fece anni di ginnastica correttiva mirata per la sua schiena e anche nuoto. Nonostante questo, la scoliosi è tuttora presente in forma significativa e gli procura spesso mal di schiena.

\section{Da genitore, non è stato sicuramente facile gestire la tua preoccupazione e cercare allo stesso tempo di essere rassicurante nei confronti di tuo figlio. Tuttavia, dal racconto della tua infanzia, noto alcune analogie con la sintomatologia dolorosa avvertita da tuo figlio. Non hai mai avuto il sospetto che i dolori alle mani e ai piedi di tuo figlio potessero essere in qualche modo collegati o simili ai dolori che tu stessa avevi riscontrato durante i tuoi episodi di febbre?}

Certamente, e di questo parlavo ogni volta con i medici che visitavano mio figlio ma la risposta era sempre la stessa: "Quando si ha la febbre tutti abbiamo dolori alle articolazioni, è normale che sia così". E quindi l'ipotesi che ci fosse una relazione tra questi indicatori, veniva subito scartata.

\section{Nonostante tutto sei riuscita a trasformare la frustrazione e le preoccupazioni in forza e tenacia, senza mai arrenderti. In che modo sei riuscita a dare un nome alla malattia che vi ha coinvolti?}

Tutto è avvenuto quando, poco più di un anno fa, ho cercato di capire se il calo di vista che mi si era presentato 
nell'ultimo periodo, fosse dovuto all'età oppure alla cornea verticillata riscontrata dagli specialisti molti anni prima. Così, in attesa di fissare una visita medica di approfondimento, sono andata sul web a cercare informazioni. Quasi subito mi sono imbattuta in una parola fino ad allora sconosciuta: Fabry.

Man mano che procedevo nella ricerca, con enorme stupore aumentava la certezza di aver finalmente trovato quello che cercavo da tanto tempo e cioè la causa di tutti $\mathrm{i}$ nostri mali. Dopo anni di frustranti visite mediche dovute alla ricerca di una cura per i disturbi che aveva mio figlio, ero sicura di aver trovato finalmente una risposta! Le spiegazioni della malattia di Anderson-Fabry trovate su internet erano esaustive e io e mio figlio rappresentavamo il quadro perfetto dei portatori di questa malattia: la cardiomiopatia ipertrofica che mi avevano diagnosticato cinque anni prima, la cornea verticillata, i dolori alle mani e ai piedi che anch'io avevo quando avevo la febbre, sommati a tutti i problemi di mio figlio, l'intolleranza al caldo e al freddo parlavano chiaro.

\section{Finalmente, dopo tante ricerche, forse avevi trovato una risposta. Ma come sai, purtroppo sul web si trovano spesso informazioni non corrette. Ti sei rivolta a qualche medico per avere un parere specialistico?}

Era il mese di febbraio del 2016. Con un po' di imbarazzo e la sensazione di sembrare presuntuosa nel proclamare la mia autodiagnosi, sono andata dal nostro medico curante, che dopo aver ascoltato le mie parole è andato a verificare le mie informazioni su uno dei suoi libroni. Con un "Brava, i vostri sintomi sono molto simili alla Fabry", ci ha invitati ad andare all'ospedale di Udine dove si trova il Centro Malattie Rare della nostra regione.

Già dalla prima visita medica fatta a Udine (dove gli angiocheratomi sulla pelle di mio figlio, davano ulteriore conferma all'ipotesi che fosse malato di Fabry), lo sguardo della dottoressa parlava chiaro sulla positività, anche se disse soltanto: "Aspettiamo le risposte dell'esame genetico".

\section{Come avete vissuto l'attesa dell'esito del test genetico?}

Speravo che l'esame genetico desse risposta positiva. In tal caso ci sarebbe stata una cura e il mio ragazzo sarebbe stato meglio. Ancora adesso ricordando quei giorni rivivo la grande emozione della speranza ma anche la rabbia che nessun medico fino ad allora avesse avuto le competenze o anche solo l'intuizione di capire che ci sono malattie poco conosciute che però fanno morire le persone dopo grandi sofferenze e tante frustrazioni. Mio figlio ha vissuto quel momento in conflitto tra la speranza e i dubbi che l'essere portatore di una malattia genetica multisistemica comporta.

\section{I vostri sentimenti sono del tutto comprensibili, perché purtroppo accomunano moltissimi pazienti che hanno ricevuto la diagnosi dopo lunghi anni di ricerche e preoccupazioni incomprese. Ma finalmente è arrivato l'esito e la prescrizione della terapia. È stato facile poter accedere alle terapie?}

Le risposte positive arrivarono dopo due mesi e mezzo, in ritardo perché in mezzo c'era agosto, periodo di ferie. La terapia enzimatica iniziammo a farla solo a fine dicembre perché il farmaco andava preparato, almeno così ci dissero. In realtà tutto si prolungò più del necessario per una questione burocratica: nessun ospedale ci voleva. Visto che il preparato dell'enzima per l'infusione è molto caro, costava troppo agli ospedali anticipare dal budget del reparto i soldi necessari per far arrivare il farmaco. Così, visto che la Fabry è una malattia multisistemica che coinvolge molti organi, $i$ vari reparti e ospedali giocavano a rimpiattino, dicendo che competeva a questo o a quel reparto e non al loro.

Ora da poco più di un anno siamo in terapia sostitutiva e facciamo un'infusione dell'enzima che non produciamo ogni due settimane. Gli esami nefrologici hanno dato risposte confortanti ma i dolori di mio figlio perdurano, perché probabilmente oramai si sono cronicizzati. Ma sono fiduciosa che prima o dopo si troverà il modo di avere un aiuto farmacologico che possa aiutare di più mio figlio. Per ora siamo già felici di sapere che i nostri organi godono del beneficio dell'enzima assunto.

\section{Riuscite a conciliare bene la terapia con gli impegni lavorativi?}

Mio figlio si è appena laureato, ha portato avanti parte degli studi in parallelo con un lavoro di letturista di contatori. Anche se gli permette di guadagnare qualcosa per mantenersi gli studi, spesso la malattia rende ingestibile questo lavoro. Infatti, con i dolori addominali quotidiani sarebbe auspicabile un lavoro più tranquillo, in un luogo che abbia un bagno sempre vicino e disponibile. Inoltre, il caldo della città già in primavera diventa insopportabile, in quanto la malattia di Fabry non permette al corpo di svolgere correttamente l'omeostasi della temperatura corporea, per non parlare dei dolori ai piedi. Insomma, non è facile. A tutto questo si aggiunge la difficoltà di ottenere il riconoscimento dei benefici della legge 104, che permetterebbe di assentarsi dal lavoro per fare le terapie. Per questo motivo, ogni due settimane mio figlio è costretto 
a prendersi una mattinata di ferie per potersi recare all'ospedale a fare la terapia enzimatica. Credo che a molti di noi pazienti con Fabry farebbe comodo poter fare la terapia domiciliare, in orari più conciliabili con il lavoro. Purtroppo, nel nostro caso la terapia domiciliare non è ancora consentita.

Ti ringrazio, Rita, per la tua disponibilità a condividere la tua esperienza nel duplice ruolo di paziente e genitore Fabry. Con la tua testimonianza, ci hai raccontato la difficoltà nell'arrivare ad una diagnosi precoce, nonostante sia tu che tuo figlio abbiate manifestato già parecchi anni fa alcuni sintomi caratteristici della malattia. Sei stata una mamma tenace e non ti sei voluta arrendere di fronte alla mancanza di risposte. Spero che la tua testimonianza sia di buon auspicio affinché la diffusione della conoscenza di questa malattia sia quanto più ampia possibile e si possa arrivare ad una diagnosi sempre più tempestiva e precoce

Ed io ringrazio voi per questa bella opportunità che mi avete dato di raccontare la nostra storia, con la speranza di trasmettere conforto e speranza per chi, come noi, vive questa esperienza. Non dimentichiamo che oggi non siamo più soli: ci sono sempre più realtà che parlano della nostra malattia. Spero che la mia storia possa aiutare a diffondere ciò che fino a pochi anni fa si ignorava.

Intervista raccolta da AIAF Onlus in collaborazione con il Comitato Editoriale 